\title{
Grammatical transformations and recognition memory of sentences
}

JAMES H. KOPLIN AND JANNAR DAVIS

VANDERBILT UNIVERSITY
A recognition memory task was adapted for the measurement of rated similarity among members of a syntactically related sentence family. A prism model of these relationships was partially supported. An additional factor (paraphrasing) was shown to contribute to significant similarity ratings. Two advantages of the recognition memory technique are (1) the active role played by $S$ in rating each sentence on a confidence scale; and (2) aural presentation.

It is obvious that there is a relationship between the sentences, "John hit the ball," and "The ball was hit by John." They are the simple declarative and passive forms of the same expression. The negative and question forms, as well as the combinations into pairs such as the negative passive, could be added. A grammar of English must be able to account for these relationships. Contemporary transformational grammars do this by assuming common elements in the derivational history of such sentences (see Chomsky, 1965, for a complete statement). It is important to point out that the rules which play a part in the derivations act only upon symbols which underlie the actual sentences of the language. The rules do not generate sentences, only the underlying structure. Psychologists, working with actual sentences, have been interested in behavioral indices of similarity as one way of confirming some properties of a transformational grammar.

Clifton \& Odom (1966) reviewed the studies which have been reported. Using a variety of methods they contributed several experiments which tested two variations on the pattern of expected sentence similarity. One was the familiar Miller cube formed by the negative, passive, and question dimensions with the kernel (simple declarative) at the intersection. The second was a model proposed by Katz and Postal which proposed differing psychological separations between the transformations, rather than the equal distances of a cube. This model can be drawn as a prism with $Q-N Q$ and $P Q-P N Q$ close together and forming the peaks (see Clifton \& Odom, Fig. 2, p. 8 and the related discussion). In general, the Katz-Postal formulation was supported.

The experiment reported below was designed to corroborate and extend the Clifton and Odom conclusions. Three major differences were incorporated: (1) The sentences were presented aurally, rather than typed on memory drum tape. This mode forced Ss to listen and to respond to each item. The added cues of inflection and intonation pattern were also present. (2) The task was one of recognition memory rather than generalization. S was asked to check a confidence rating scale after listening to each sentence as an indication of the presence or absence of that sentence earlier in the list. This is an adaptation of a task used by Underwood (1965) to study mediation effects. (3) One additional type of sentence was included. These sentences were related in a manner not represented in either the cube or prism model, and the application of the simple transformation rules could not lead from one sentence of a pair to the other. They are paraphrases such as, "John strikes me as pompous," and "I regard John as pompous," (Chomsky, 1965, p. 162). Items such as these tested the influence of factors other than transformational relatedness as a basis for judged similarity of sentences.

This experiment did not investigate all possible combinations of the eight sentence types found in the model. To provide the greatest discrepancy between the cube and prism models, four types of pairs were chosen: $K-N, P-K, Q-P Q, Q N-Q$. The letters refer to kernel, negative, passive, and question. The paraphrase group formed a fifth type labeled $X-Y$.

\section{Method}

Two lists (A and B) of 125 sentences each were constructed. Section 1 of list A, the first 60 sentences, contained the 20 stimulus sentences (four entries for each of the five types listed above), 30 filler sentences, and 10 repeated filler sentences. Section 2 , the remaining 65 items, contained 20 transformations (if the $\mathrm{K}$ form appeared in Section 1, the $\mathrm{N}$ form appeared here, etc.), 28 filler sentences, and 17 repeated filler sentences. The fillers contained an approximately equal number of each of the transformation types used in the experimental sentences. List B was constructed from the sameitems as List $A$ except that the transformations on the items were randomly reassigned. A list pair assigned to the $\mathrm{K}-\mathrm{N}$ type in List $\mathrm{A}$ might appear as a $\mathrm{QN}-\mathrm{Q}$ type in List B.

The two sections of List A were randomized separately with the restriction that the mean number of items appearing between a stimulus sentence in Section 1 and the corresponding transformation in Section 2 be approximately equal (Range $=54$ to 59 ). The 125 sentences of List A were recorded on tape with an identification number preceding each item and an inter-item interval of $5 \mathrm{sec}$. List B was prepared in the same manner with the appropriate changes in item content.

Four independent groups of $\mathrm{Ss}(\mathrm{N}=17$ in each) participated in the experiment. Two groups heard each of the two lists. E read instructions asking $S$ to indicate immediately after hearing each sentence his degree of certainty that he had heard the sentence before in the list. Note that this decision was requested beginning with the first 
Table 1. Mean Scale Rating of Sentence Types

\begin{tabular}{lccc} 
Sentence Type & List A & List B & Combined A\& B \\
\hline 1. Repeat & 5.70 & 6.03 & 5.87 \\
2. QN-Q & 4.85 & 4.84 & 4.84 \\
3. P-K & 3.80 & 4.56 & 4.18 \\
4. Q-PQ & 3.46 & 4.22 & 3.84 \\
5. K-N & 3.46 & 3.05 & 3.25 \\
6. X-Y & 2.81 & 3.22 & 3.02 \\
7. Control & 1.49 & 1.50 & 1.50 \\
\hline
\end{tabular}

sentence of Section 1. There was no pause or change of instructions throughout the complete list. Responses were recorded on numbered 7-point confidence scales provided on the answer sheet. The scales ranged from position 1, "Positive, this sentence has NOT been presented before," through position 7 , "Positive, this sentence HAS been presented before."

Results

Mean confidence scale ratings were computed for each $S$ for the second member of each of the five types of experimental pairs and also for the repeated and control sentences. The mean scale values for all Ss responding to List $A$ and List $B$ and for the combined lists are presented in Table 1 . On this scale, confidence that a sentence was in fact heard before in the list is expressed as a large scale value (Maximum $=7$ ). Conversely, a new sentence will have a small scale value (Minimum = 1).

An analysis of variance was performed using the Between-S variables of Lists (A or B), and Groups ( 1 or $2)$, plus the Within-S variables of Sentence Type $(\mathrm{K}-\mathrm{N}$, $P-K$, etc.). The main effect of Sentence Type $(F=111.52$, $\mathrm{df}=6 / 384, \mathrm{p}<.001$ ) and the Sentence Type by List interaction $(F=2.63, \mathrm{df}=6 / 384, \mathrm{p}<.05)$ were significant sources of variance. Since Groups produced no significant effects, the data were collapsed over this factor. Because of the significant interaction, a Newman-Keuls multiple comparison of means was performed on the separate lists. For List A $(p<.05)$ all means not underlined by the same line are significantly different: $12 \underline{3} 4567$. For List $B(p<.05)$ the grouping is: $1 \underline{2} 3 \begin{array}{lllll}5 & 6 & 7 .\end{array}$

\section{Discussion}

The efficiency of the recognition memory technique was demonstrated by the Sentence Types at the extremes in Table 1 . In both lists repeated sentences were correctly judged as having been heard before, as shown by the confidence scale rating significantly higher than that for all other types. Similarly, Control sentences appearing in the list for the first time were accurately judged as such in both lists. This "false alarm" rate is unusually low and changed very little throughout the presentation of the 125 sentences. The Sentence Types
(Categories 2-5 in Table 1) to be compared to the Clifton \& Odom (1966) results provided different outcomes in the two lists. List A gave results consistent with the prism model. The $Q N-Q$ pairs were judged significantly more similar than the three sentence types representing the base and sides of the prism, which were not differentiated by the confidence scale ratings. Such consistency was not to be found in List $B$. The means are properly ordered, but the $Q N-Q$ Type was not significantly different from the P-K and Q-PQ sentences. Further, the K-N Type was significantly lower than the other three categories.

There is no ready explanation for the list differences. It is interesting to note that List A was the list originally prepared for the experiment while List $B$ was derived from List $A$ by rewriting the sentence content in a new syntactic form. This suggests that the original list may have incorporated subtle semantic features which did not "translate" well. Clifton \& Odom (1966) also took note of this problem.

Examination of the $X-Y$ or paraphrase sentences in List A showed that they were significantly different from syntactically related pairs and from control pairs. This indicated that judged similarity may be based on relationships other than that of syntactic family membership and that this relatedness was less easily recognized. Again, there was a problem with List B. Here the X-Y Type was not significantly different from the $K-N$ sentences. The comment made above seems to apply here as well. The X-Y items used in List A were selected from examples presented in Chomsky (1965). In List B the paraphrases were derived from sentences randomly selected from the experimental sentences of List A. Perhaps a skilled linguist trained in the application of transformational rules could point out a relationship between the two items of the pairs which had been overlooked.

This exploratory study has shown the effectiveness of a recognition memory approach to the study of sentence similarity. Its chief methodological virtue is that S must listen to each item and respond immediately using a sensitive confidence rating measure. Limited corroboration has been provided for the Clifton \& Odom (1966) version of the Katz-Postal prism model. Finally, the importance in tasks of this type, of factors other than syntactic relatedness has been demonstrated.

\section{References}

Chomsky, N. Aspects of the theory of syntax. Cambridge: The MIT Press, 1965.

Clifton, C., Jr., \& Odom, Penelope. Similarity relations among certain English sentence constructions. Psychol. Monogr., 1966, 80 , No. 5 (Whole No. 613).

Underwood, B. J. False recognition produced by implicit verbal responses. J. exp. Psychol., 1965, 1, 122-129. 